\title{
Classification of solvable Lie algebras
}

\author{
W. A. de Graaf, \\ School of Mathematics and Statistics, \\ University of Sydney, \\ Australia \\ email: wdg@maths.usyd.edu.au
}

\begin{abstract}
In this paper we illustrate some simple ideas that can be used for obtaining a classification of small-dimensional solvable Lie algebras. Using these we obtain the classification of 3 and 4 dimensional solvable Lie algebras (over fields of any characteristic). Precise conditions for isomorphism are given.
\end{abstract}

Solvable Lie algebras have been classified by G. M. Mubarakzjanov (upto dimension 6 over a real field, [8], [7], see also [9]), and by J. Patera and H. Zassenhaus (upto dimension 4 over any perfect field, [10]). In this paper we explore the possibility of using the computer to obtain a classification of solvable Lie algebras. The possible advantages of this are clear. The problem of classifying Lie algebras needs a systematic approach, and the more the computer is invloved, the more systematic the methods have to be. However, the drawback is that the computer can only handle finite data. For example, below we will consider orbits of the action of the automorphism group of a Lie algebra on the algebra of its derivations. Now, if the ground field is infinite, then I know of no algorithm for obtaining these orbits. In our approach we use the computer (specifically the technique of Gröbner bases) to decide isomorphism of Lie algebras, and to obtain explicit isomorphisms if they exist.

The procedure that we use to classify solvable Lie algebras is based on some simple ideas, which are described in Section 1 (and for which we do not claim any originality). Then in Section 2 we describe the use of Gröbner bases. In Section [3 solvable Lie algebras of dimension 3 are classified. In Section 4 the same is done for dimension 4 . We show that our classification in dimension 4 differs slightly from the one found in [10] (i.e., we find a few more Lie algebras).

For doing the explicit calculations reported here we have used the computer algebra system Magma ([2]).

\section{General lemmas}

In the sequel we denote the field we work with by $F$. 
Lemma 1 Let $L$ be a solvable Lie algebra. Then there is a subalgebra $K \subset L$ of codimension 1, and a derivation $d$ of $K$ such that $L=F x_{d} \oplus K$. Here $\left[x_{d}, y\right]=d(y)$ for all $y \in K$. Moreover, if $L$ is not Abelian, then $d$ and $K$ can be chosen such that $d$ is an outer derivation of $K$.

Proof. Let $K$ be any subspace of codimension 1 containing $[L, L]$, and let $x \in L$ span a complementary subspace. Then $K$ is an ideal of $L$ and we get the result with $d=\operatorname{ad} x$ and $x_{d}=x$.

The proof of the second statement is by induction on $\operatorname{dim} L$. If $\operatorname{dim} L=2$ then the statement is clear. Now suppose $\operatorname{dim} L=n>2$ and write $L=F y \oplus K$. Suppose that $\operatorname{ad} y$ is an inner derivation of $K$, i.e., $\operatorname{ad} y=\operatorname{ad} u$ for some $u \in K$. Set $z=y-u$; then $L=K \oplus F z$ and $[z, K]=0$. So $K$ is non-Abelian and by induction we have $K=F x \oplus K_{1}$, where $\left.\operatorname{ad} x\right|_{K_{1}}$ is an outer derivation. Set $K_{2}=K_{1} \oplus F z$, then also ad $\left.x\right|_{K_{2}}$ is an outer derivation, and $L=F x \oplus K_{2}$.

Lemma 2 Let $K$ be a solvable Lie algebra and $d_{1}, d_{2}$ derivations of $K$. Set $L_{i}=F x_{d_{i}} \oplus K$, $i=1,2$. Suppose that there is an automorphism $\sigma$ of $K$ such that $\sigma d_{1} \sigma^{-1}=\lambda d_{2}$, for some scalar $\lambda \neq 0$. Then $L_{1}$ and $L_{2}$ are isomorphic.

Proof. Define a linear map $\tilde{\sigma}: L_{1} \rightarrow L_{2}$ by $\tilde{\sigma}(y)=\sigma(y)$ for $y \in K$ and $\tilde{\sigma}\left(x_{d_{1}}\right)=\lambda x_{d_{2}}$. Then $\tilde{\sigma}$ is a bijective linear map. The fact that it is an isomorphism can be established by direct verification.

The procedure based on these lemmas is as follows. Let $K$ be a solvable Lie algebra of dimension $n$. We compute the automorphism group $\operatorname{Aut}(K)$ of $K$ and the derivation algebra $\operatorname{Der}(K)$ of $K$. We denote the subalgebra of inner derivations by $\operatorname{Inn}(K)$. It is straightforward to see that Lie algebras defined by derivations in the same coset of $\operatorname{Inn}(K)$ in $\operatorname{Der}(K)$ are isomorphic. Now the group $G(K)=F^{*} \times \operatorname{Aut}(K)$ acts on the cosets $d+\operatorname{Inn}(K)$ for $d \in \operatorname{Der}(K)$ by $(\lambda, \sigma) \cdot d+\operatorname{Inn}(K)=\lambda \sigma d \sigma^{-1}+\operatorname{Inn}(K)$. We compute orbit representatives of the action of $G(K)$ on $\operatorname{Der}(K) / \operatorname{Inn}(K)$. For every such representative we get a solvable Lie algebra of dimension $n+1$. Subsequently we weed out the isomorphic ones.

When doing this we often deal with Lie algebras given by a multiplication table containing parameters. An easy trick that often works to reduce the number of parameters is to consider a diagonal base change. Let $\left\{x_{1}, \ldots, x_{n}\right\}$ be a basis of $L$, and set $y_{i}=\alpha_{i} x_{i}$. Then write down the multiplication table of $L$ with respect to the $y_{i}$. Often it is possible to choose the $\alpha_{i}$ in such a way that we can get rid of one or more parameters.

When $K$ is Abelian of dimension $n$ we have that $\operatorname{Der}(K)=M_{n}(F)$ and $\operatorname{Aut}(K)=$ $\mathrm{GL}(n, F)$. In this case representatives of the orbits of $\operatorname{Aut}(K)$ are known, by the following well-known theorem (for a proof we refer to [6]).

Theorem 3 Let $A$ be an $n \times n$-matrix over a field $F$. Then $A$ is similar over $F$ to a unique block-diagonal matrix, containing the blocks $C\left(f_{1}\right), \ldots, C\left(f_{s}\right)$ where $C\left(f_{k}\right)$ is the companion matrix of the non-constant monic polynomial $f_{k}$, and $f_{k} \mid f_{k+1}$ for $1 \leq k \leq s-1$. 
The unique block-diagonal matrix is called the rational canonical form of $A$.

We end this section with some remarks on notational conventions that we use. In this paper we usually describe an $n$-dimensional Lie algebra by giving its multiplication table with respect to a basis, which on most occasions is denoted $x_{1}, \ldots, x_{n}$. In these multiplication tables we use the convention that products which are not listed are zero. Also when representing a linear map by a matrix we always use the column convention.

\section{Constructing isomorphisms}

One of the main problems when classifying Lie algebras is to decide whether two of them are isomorphic. A very convenient tool for doing that is Gröbner bases (cf. [4]). (For an introduction into Gröbner bases we refer to [3].) By way of example we describe how this works.

Consider the 3 -dimensional Lie algebra $L_{1}$ with basis $x_{1}, x_{2}, x_{3}$ and multiplication table

$$
\left[x_{1}, x_{2}\right]=x_{2}, \quad\left[x_{1}, x_{3}\right]=a x_{3},
$$

and the 3 -dimensional Lie algebra $L_{2}$ with basis $y_{1}, y_{2}, y_{3}$ and multiplication table

$$
\left[y_{3}, y_{1}\right]=y_{2},\left[y_{3}, y_{2}\right]=b y_{1}+y_{2}
$$

The question is whether $L_{1}$ and $L_{2}$ are isomorphic, and if so for which values of $a, b$. In that case we would also like to have an explicit isomorphism. An isomorphism will map the nilradical of $L_{1}$ onto the nilradical of $L_{2}$. So an isomorphism $\phi: L_{1} \rightarrow L_{2}$ has the form $\phi\left(x_{1}\right)=a_{1} y_{1}+a_{2} y_{2}+a_{3} y_{3}, \phi\left(x_{2}\right)=b_{1} y_{1}+b_{2} y_{2}, \phi\left(x_{3}\right)=c_{1} y_{1}+c_{2} y_{2}$. Now this is an isomorphism if and only if the following polynomial equations are satisfied

$$
b a_{3} b_{2}-b_{1}=0, a_{3} b_{1}+a_{3} b_{2}-b_{2}=0, b a_{3} c_{2}-a c_{1}=0, a_{3} c_{1}+a_{3} c_{2}-a c_{2}=0,
$$

and

$$
D_{1} a_{3}-1=0, D_{2}\left(b_{1} c_{2}-b_{2} c_{1}\right)-1=0 .
$$

The last two equations are added to ensure that the determinant is nonzero. Now in Magma we compute a Gröbner basis of the ideal of $\mathbb{Q}\left[D_{1}, D_{2}, a_{1}, a_{2}, a_{3}, b_{1}, b_{2}, c_{1}, c_{2}, a, b\right]$ generated by the left hand sides of these equations. We use the lexicographical ordering. This leads to a Gröbner basis with a triangular structure, which on many occasions makes it possible to find an explicit solution. Also, we let $a, b$ be the smallest variables in the ordering; this makes it likely that the Gröbner basis contains polynomials in only $a$ and $b$ (cf. [3], Chapter 3, Theorem 2). From these we can derive necessary conditions for isomorphism. So in Magma we do the following

\footnotetext{
$>\mathrm{P}<\mathrm{D} 1, \mathrm{D} 2, \mathrm{a} 1, \mathrm{a} 2, \mathrm{a} 3, \mathrm{~b} 1, \mathrm{~b} 2, \mathrm{c} 1, \mathrm{c} 2, \mathrm{a}, \mathrm{b}>:=\operatorname{PolynomialRing}(\operatorname{Rationals}(), 11)$;

$>\mathrm{r}:=[\mathrm{b} * \mathrm{a} 3 * \mathrm{~b} 2-\mathrm{b} 1, \mathrm{a} 3 * \mathrm{~b} 1+\mathrm{a} 3 * \mathrm{~b} 2-\mathrm{b} 2, \mathrm{~b} * \mathrm{a} 3 * \mathrm{c} 2-\mathrm{a} * \mathrm{c} 1, \mathrm{a} 3 * \mathrm{c} 1+\mathrm{a} 3 * \mathrm{c} 2-\mathrm{a} * \mathrm{c} 2$,

$>\mathrm{D} 1 * \mathrm{a} 3-1, \mathrm{D} 2 *(\mathrm{~b} 1 * \mathrm{c} 2-\mathrm{b} 2 * \mathrm{c} 1)-1]$;
} 


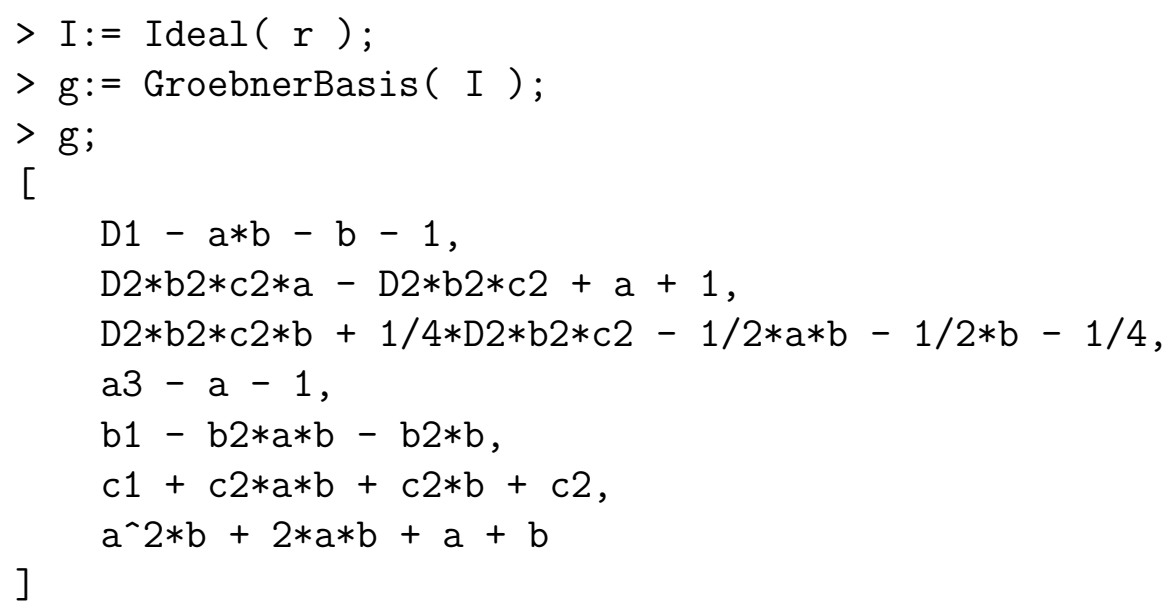

From the last equation we get

$$
b=-\frac{a}{(a+1)^{2}} .
$$

From this we also see that the algebras are not isomorphic if $a=-1$. Solutions to the fourth, fifth and sixth equations are easily found, e.g., $a_{1}=a_{2}=0, a_{3}=a+1, b_{1}=a b+b$, $b_{2}=1, c_{1}=a b+b+1, c_{2}=-1$. By direct verification we get that this indeed defines an isomorphism, if (11) holds, and $a \neq 1$ (otherwise the determinant is zero). If $a=1$ then by a separate calculation we get that the Gröbner basis is $\{1\}$. So in that case $L_{1}$ and $L_{2}$ are not isomorphic. The conclusion is that $L_{1} \cong L_{2}$ if and only if (11) and $a \neq 1$. Moreover, in that case we also have an explicit isomorphism.

In the above discussion we have taken the ground field to be $\mathbb{Q}$. However, the conclusion holds over any field of characteristic 0, since over any such field the Gröbner basis will be the same. We can also easily reach the same conclusion for any field of characteristic $p>0$. For that we note that the input polynomials $g_{i}$ are defined over any field. Now by using the Magma function Coordinates we can find polynomials $p_{i}$ such that $\sum p_{i} g_{i}=a^{2} b+2 a b+a+b$. The coefficients of the $p_{i}$ are rational numbers. So from these coefficients we find a finite set of characteristics over which they are not defined. We then have to do the computation separately over fields of those characteristics. Over all other fields we have that the ideal generated by the input polynomials contains $a^{2} b+2 a b+a+b$. Hence for all those fields we reach the same conclusion (since the explicit isomorphism is defined over any field). In our example we have

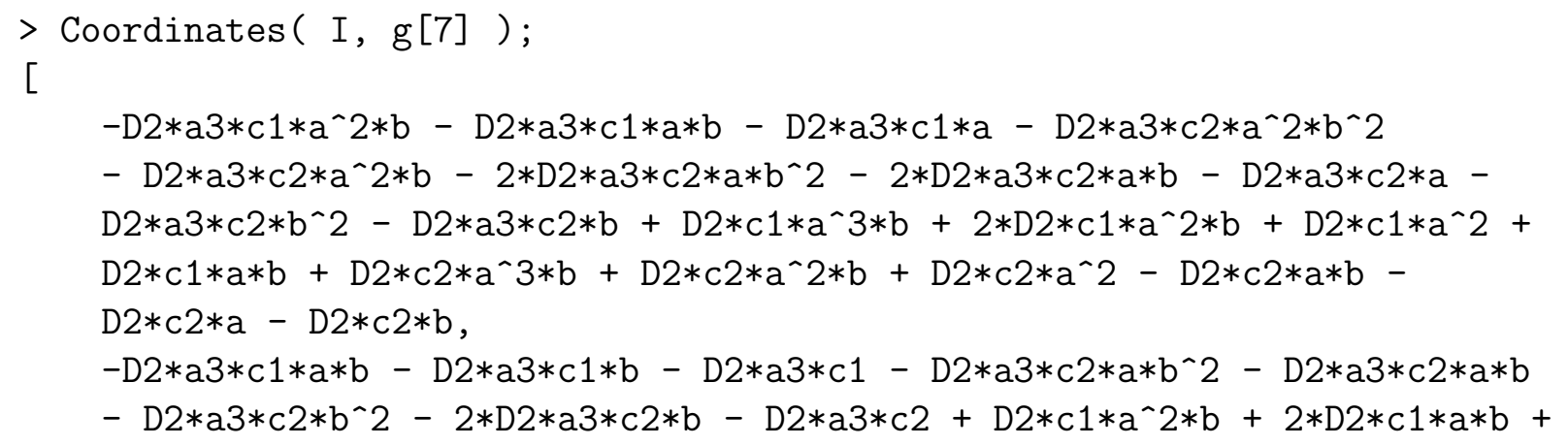




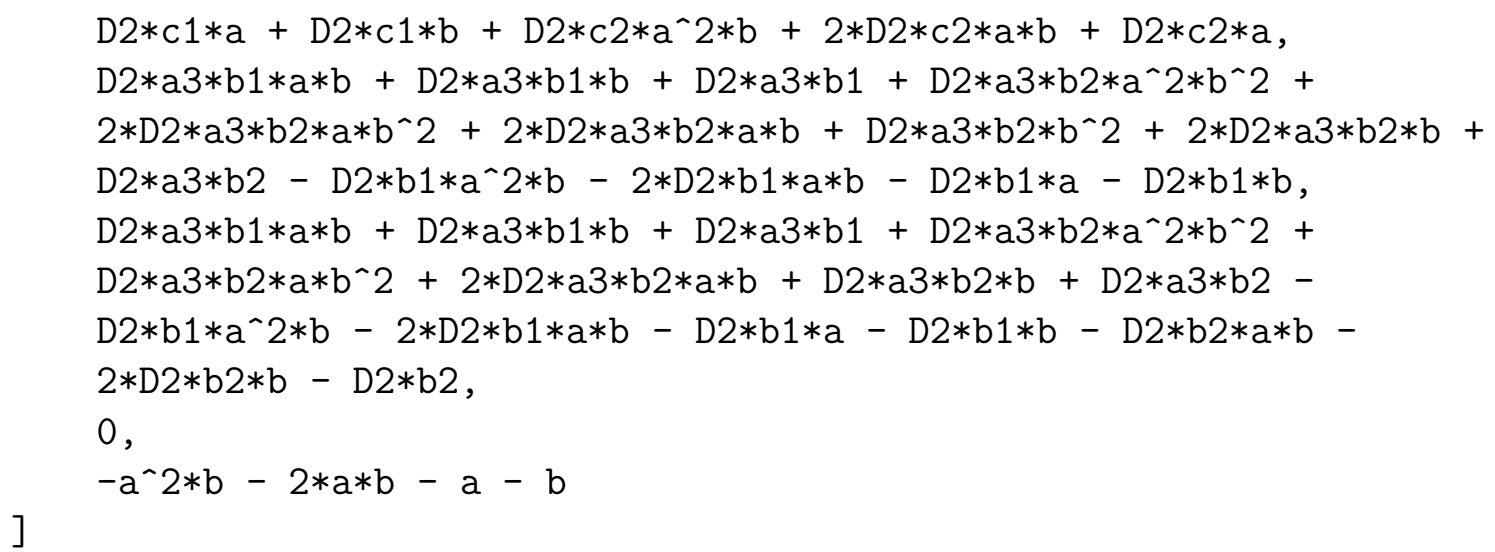

The coefficients of these polynomials are all integers. Hence the above conclusion holds for all fields.

\section{The 3-dimensional case}

There are only two (isomorphism classes of) Lie algebras of dimension 2 .

First we consider the Lie algebra $K$ spanned by $x_{1}, x_{2}$ with $\left[x_{1}, x_{2}\right]=0$. Then $\operatorname{Aut}(K)=$ $\mathrm{GL}(2, F)$, and $\operatorname{Der}(K)=M_{2}(F)$ (i.e., the space of all $2 \times 2$-matrices). In this case the rational canonical form of an element in $\operatorname{Der}(K)$ is either

$$
\lambda\left(\begin{array}{ll}
1 & 0 \\
0 & 1
\end{array}\right),
$$

or

$$
\left(\begin{array}{ll}
0 & a \\
1 & b
\end{array}\right)
$$

Fist of all, $\lambda=0$ gives a Lie algebra that is the three dimensional Abelian Lie algebra. If $\lambda \neq 0$ then by Lemma 2 we may divide by $\lambda$ and get the Lie algebra $L^{2}$ spanned by $x_{1}, x_{2}, x_{3}$ and nontrivial brackets $\left[x_{3}, x_{1}\right]=x_{1},\left[x_{3}, x_{2}\right]=x_{2}$. In the third case we get the Lie algebras $L_{a, b}$ spanned by $x_{1}, x_{2}, x_{3}$ and multiplication table

$$
\left[x_{3}, x_{1}\right]=x_{2},\left[x_{3}, x_{2}\right]=a x_{1}+b x_{2} .
$$

From a Gröbner basis computation we get that $L_{a, b} \cong L_{c, d}$ implies $a d^{2}-b^{2} c=0$ and $c_{3}^{2} c-a=0$, for some nonzero $c_{3} \in F$. Furthermore, this holds over any field since the coordinates of these polynomials (with respect to the input basis) all have integer coefficients.

Also by applying a diagonal base change we see that $L_{a, b} \cong L_{\alpha^{2} a, \alpha b}$ for $\alpha \neq 0$ (the base change is $\left.y_{1}=x_{1}, y_{2}=\alpha^{-1} x_{2} y_{3}=\alpha^{-1} x_{3}\right)$. Now we distinguish two cases:

1. $b \neq 0$. Then $L_{a, b} \cong L_{a, 1}$. So we get the class of Lie algebras $L_{a}^{3}=L_{a, 1}$. The above discussion implies that $L_{a}^{3} \cong L_{b}^{3}$ if and only if $a=b$. 
2. $b=0$. We get the class of Lie algebras $L_{a}^{4}=L_{a, 0}$. In this case $L_{a} \cong L_{b}$ if and only if $a=\alpha^{2} b$ for some nonzero $\alpha \in F$.

The Lie algebras $L_{a}^{3}$ and $L_{b}^{4}$ are never isomorphic. This can be established by a Gröbner basis computation. It can also be shown in the following way. Suppose that $L_{a}^{3} \cong L_{c}^{4}$. Then $a d^{2}-b^{2} c=0$ amounts to $c=0$. However, $L_{0}^{4}$ is nilpotent, and $L_{a}^{3}$ is not.

The other 2-dimensional Lie algebra (with basis $x_{1}, x_{2}$ and $\left[x_{1}, x_{2}\right]=x_{2}$ ) does not have to be considered, as it has no outer derivations.

Summarising we get the following solvable Lie algebras of dimension 3 :

$$
\begin{aligned}
& L^{1}\left[x_{3}, x_{1}\right]=0,\left[x_{3}, x_{2}\right]=0,\left[x_{1}, x_{2}\right]=0 . \\
& L^{2}\left[x_{3}, x_{1}\right]=x_{1},\left[x_{3}, x_{2}\right]=x_{2},\left[x_{1}, x_{2}\right]=0 . \\
& L_{a}^{3}\left[x_{3}, x_{1}\right]=x_{2},\left[x_{3}, x_{2}\right]=a x_{1}+x_{2},\left[x_{1}, x_{2}\right]=0 . \\
& L_{a}^{4}\left[x_{3}, x_{1}\right]=x_{2},\left[x_{3}, x_{2}\right]=a x_{1},\left[x_{1}, x_{2}\right]=0 .
\end{aligned}
$$

Here the only isomorphisms are $L_{a}^{4} \cong L_{b}^{4}$ if and only if $a=\alpha^{2} b$. I have established the non-isomorphism of $L^{2}$ with $L_{a}^{3}, L_{a}^{4}$ by Gröbner basis calculations.

We count the number of non-isomorphic solvable Lie algebras over the finite field with $q$ elements. The classes $L^{1}, L^{2}, L^{3}$ always give $q+2$ Lie algebras. If the characteristic of the ground field is not 2 , then $L_{a}^{4}$ gives 3 more Lie algebras. In that case the total number is $q+5$. If the characteristic is 2 , then all elements of $\mathbb{F}_{q}$ are squares, meaning that we get two isomorphism classes of Lie algebras (i.e., $L_{0}^{4}$ and $L_{1}^{4}$ ). In that case the total number is $q+4$.

Remark. Our classification is the same as the one obtained in [10]. More precisely, we have $L_{3,1} \cong L^{1}, L_{3,2} \cong L_{0}^{4}, L_{3,3} \cong L_{0}^{3}, L_{3,4} \cong L^{2}, L_{3,5} \cong L_{\alpha}^{4}$ (where $\alpha$ is as in [10]), $L_{3,6} \cong L_{-\alpha}^{3}$, and $L_{3,7} \cong L_{-1 / 4}^{3}$ (if the characteristic is not 2) and $L_{3,7} \cong L_{1}^{4}$ (if the characteristic is 2).

So we have the same classification, but with a shorter description.

Remark. From the method used we get an easy algorithm for recognising a given 3dimensional Lie algebra $K$ as one of the $L^{i}$. First we find a 2-dimensional Abelian ideal. Let $x$ span a complement to this ideal. Then we find the rational canonical form of the adjoint action of $x$ on the ideal. From this we immediately see to which algebra $K$ is isomorphic.

\section{The 4-dimensional case}

Here we have to find derivation algebras, automorphism groups of 3-dimensional Lie algebras $K$. The algebras that will appear in the final classification will be denoted $M^{i}$.

First we consider $K=L^{1}$. This Lie algebra is Abelian, so the orbits of the derivations under the action of $\operatorname{Aut}(K)$ are given by the rational canonical form of matrices. If this form 
consists of three $1 \times 1$ blocks, then because of the divisibility condition in Theorem 3 , they have to be the same. After division we get two algebras: the 4-dimensional commutative algebra (denoted by $M^{1}$ ), and

$$
M^{2}: \quad\left[x_{4}, x_{1}\right]=x_{1},\left[x_{4}, x_{2}\right]=x_{2},\left[x_{4}, x_{3}\right]=x_{3} .
$$

If there is a $1 \times 1$-block and a $2 \times 2$-block, then again because of divisibility we have

$$
D=\left(\begin{array}{ccc}
s & 0 & 0 \\
0 & 0 & -s t \\
0 & 1 & s+t
\end{array}\right)
$$

Denote the corresponding Lie algebra by $K_{s, t}$. After multiplying $x_{4}, x_{3}$ by $\alpha$ (and $x_{1}, x_{2}$ by 1 ) we see that this Lie algebra is isomorphic to $K_{\alpha s, \alpha t}$, where $\alpha \neq 0$. We consider a few cases.

First $s \neq 0$. Then we can take $\alpha=s^{-1}$, and we get the Lie algebras

$$
M_{a}^{3}: \quad\left[x_{4}, x_{1}\right]=x_{1},\left[x_{4}, x_{2}\right]=x_{3},\left[x_{4}, x_{3}\right]=-a x_{2}+(a+1) x_{3} .
$$

Gröbner basis computations reveal that $M_{a}^{3} \cong M_{b}^{3}$ if and only if $a=b$.

If $s=0$, then if $t \neq 0$, we can take $\alpha=t^{-1}$, and get

$$
M^{4}: \quad\left[x_{4}, x_{2}\right]=x_{3},\left[x_{4}, x_{3}\right]=x_{3} .
$$

Finally, if $s=t=0$ we get

$$
M^{5}: \quad\left[x_{4}, x_{2}\right]=x_{3} .
$$

If there is a $3 \times 3$-block in the rational normal form, then we get the Lie algebras $K_{s, t, u}$ :

$$
\left[x_{4}, x_{1}\right]=x_{2},\left[x_{4}, x_{2}\right]=x_{3},\left[x_{4}, x_{3}\right]=s x_{1}+t x_{2}+u x_{3} .
$$

Multiplying $x_{2}, x_{3}, x_{4}$ by $\alpha, \alpha^{2}, \alpha$ respectively, we see that $K_{s, t, u} \cong K_{\alpha^{3} s, \alpha^{2} t, \alpha u}$. Hence, if $u \neq 0$, then we can take $\alpha=u^{-1}$, and we get the Lie algebras

$$
M_{a, b}^{6}: \quad\left[x_{4}, x_{1}\right]=x_{2},\left[x_{4}, x_{2}\right]=x_{3},\left[x_{4}, x_{3}\right]=a x_{1}+b x_{2}+x_{3} .
$$

A Gröbner basis computation shows that $M_{a, b}^{6} \cong M_{c, d}^{6}$ if and only if $a=c$ and $b=d$.

If $u=0$ then we get the Lie algebras

$$
M_{a, b}^{7}: \quad\left[x_{4}, x_{1}\right]=x_{2},\left[x_{4}, x_{2}\right]=x_{3},\left[x_{4}, x_{3}\right]=a x_{1}+b x_{2} .
$$

From the above discussion we see that $M_{a, b}^{7} \cong M_{c, d}^{7}$ if $a=\alpha^{3} c$ and $b=\alpha^{2} d$ (for some $\alpha \neq 0$ ). From a Gröbner basis computation we have that this is also a necessary condition. So, if both parameters are nonzero, than by a suitable choice for $\alpha$ we can make them equal. Hence this class splits into three subclasses: $M_{a, a}^{7}, M_{a, 0}^{7}, M_{0, b}^{7}$. Among the first class there are no isomorphisms. 
Now we consider $K=L^{2}$. The coset representatives of the outer derivations of $K$ (modulo inner derivations) are

$$
D=\left(\begin{array}{lll}
s & t & 0 \\
u & 0 & 0 \\
0 & 0 & 0
\end{array}\right)
$$

The automorphism group of $K$ consists of the elements

$$
\phi=\left(\begin{array}{ccc}
\alpha & \beta & \epsilon_{1} \\
\gamma & \delta & \epsilon_{2} \\
0 & 0 & 1
\end{array}\right)
$$

where $\alpha \delta-\beta \gamma \neq 0$. Modulo inner derivations we have

$$
(\operatorname{det} \phi) \phi D \phi^{-1}=\left(\begin{array}{ccc}
s \alpha \delta-t \alpha \gamma+u \beta \delta & -s \alpha \beta+t \alpha^{2}-u \beta^{2} & 0 \\
s \gamma \delta-t \gamma^{2}+u \delta^{2} & -s \beta \gamma+t \alpha \gamma-u \beta \delta & 0 \\
0 & 0 & 0
\end{array}\right)
$$

First we suppose that $s \neq 0$. Then we choose $\beta=\gamma=0$ and $\alpha=1 / s, \delta=1$. Then $D$ is conjugate to

$$
\left(\begin{array}{lll}
1 & w & 0 \\
v & 0 & 0 \\
0 & 0 & 0
\end{array}\right)
$$

for some $v, w$. This leads to the Lie algebras $K_{v, w}$ with basis $x_{1}, x_{2}, x_{3}, x_{4}$ and nonzero commutators

$$
\left[x_{4}, x_{1}\right]=x_{1}+v x_{2},\left[x_{4}, x_{2}\right]=w x_{1},\left[x_{3}, x_{1}\right]=x_{1},\left[x_{3}, x_{2}\right]=x_{2} .
$$

Suppose that $w \neq 0$, then by setting $y_{1}=w x_{1}, y_{i}=x_{i}$ for $i=2,3,4$, we see that $K_{v, w} \cong K_{v^{\prime}, 1}$. Denote this Lie algebra simply by $K_{v}$. By some calculations it is seen that the centralizer $C\left(\operatorname{ad} K_{v}\right)$ in the full (associative) matrix algebra is spanned by the identity and

$$
\left(\begin{array}{llll}
1 & 1 & 0 & 0 \\
v & 0 & 0 & 0 \\
0 & 0 & 0 & v \\
0 & 0 & 1 & 1
\end{array}\right) .
$$

The minimal polynomial of this last matrix is $T^{2}-T-v$. Suppose that the characteristic of $F$ is not 2 . Then, if $v=-\frac{1}{4}$, this algebra has a nonzero radical. We get the Lie algebra

$$
N: \quad\left[x_{4}, x_{1}\right]=x_{1}-\frac{1}{4} x_{2},\left[x_{4}, x_{2}\right]=x_{1},\left[x_{3}, x_{1}\right]=x_{1},\left[x_{3}, x_{2}\right]=x_{2} .
$$

(We denote this algebra by $N$ and not by $M^{8}$, because it is isomorphic to a Lie algebra that we define later). On the other hand, if $v \neq-\frac{1}{4}$ then $C\left(\operatorname{ad} K_{v}\right)$ is semisimple. Also, if $T^{2}-T-v$ has a root in the base field, then it splits. This implies that $K_{v}$ is isomorphic 
to the direct sum of two 2-dimensional Lie algebras (namely the non-commutative ones) (cf. [5], §1.15, 11]). We get the Lie algebra

$$
M^{8}: \quad\left[x_{1}, x_{2}\right]=x_{2},\left[x_{3}, x_{4}\right]=x_{4} .
$$

Now suppose that $T^{2}-T-v$ does not have a root in $F$. Then $K_{v}$ is indecomposable. Suppose that $K_{v} \cong K_{w}$, where also $T^{2}-T-w$ has no root in $F$. Then from the Gröbner basis it follows that $v+\frac{1}{4}=\alpha^{2}\left(w+\frac{1}{4}\right)$ for some nonzero $\alpha \in F$. (There is also another argument to prove this: as seen above $K_{v}$ splits over $F(\sqrt{1+4 v})$ so also $K_{w}$ splits over this field. Hence $\sqrt{1+4 w} \in F(\sqrt{1+4 v})$. This implies the claim.) Conversely, suppose that $v+\frac{1}{4}=\alpha^{2}\left(w+\frac{1}{4}\right)$ for some nonzero $\alpha \in F$. Let $\phi: K_{v} \rightarrow K_{w}$ be the linear map given by $\phi\left(x_{1}\right)=\alpha y_{1}+\frac{1}{2}(1-\alpha) y_{2}, \phi\left(y_{2}\right)=y_{2}, \phi\left(y_{3}\right)=y_{3}, \phi\left(x_{4}\right)=\frac{1}{2}(1-\alpha) y_{3}+\alpha y_{4}$. Then $\phi$ is an isomorphism. We conclude that $K_{v} \cong K_{w}$ if and only if $v+\frac{1}{4}=\alpha^{2}\left(w+\frac{1}{4}\right)$ for some nonzero $\alpha \in F$.

Now we deal with the case where the characteristic of $F$ is 2 . Just as above, if $T^{2}+T+a$ factors over $F$, then $K_{v}$ is isomorphic to a direct sum. If the polynomial does not factor, then $K_{v}$ is indecomposable. From the Gröbner basis computation it follows that $K_{v} \cong K_{w}$ implies that $X^{2}+X+v+w$ has roots in $F$. Conversely, suppose that this equation has a root $\alpha \in F$. Then there is an isomorphism $\phi: K_{v} \rightarrow K_{w}$ given by $\phi\left(x_{1}\right)=y_{1}+\alpha y_{2}$, $\phi\left(x_{2}\right)=y_{2}, \phi\left(x_{3}\right)=y_{3}, \phi\left(x_{4}\right)=\alpha y_{3}+y_{4}$. So $K_{v} \cong K_{w}$ if and only if $X^{2}+X+v+w$ has roots in $F$. The conclusion is that we get the Lie algebras

$$
M_{a}^{9}: \quad\left[x_{4}, x_{1}\right]=x_{1}+a x_{2},\left[x_{4}, x_{2}\right]=x_{1},\left[x_{3}, x_{1}\right]=x_{1},\left[x_{3}, x_{2}\right]=x_{2},
$$

where $a \in F$ is such that $T^{2}-T-a$ has no root in the base field.

Now suppose that $w=0$. Then $K_{v, 0}$ is the direct sum of ideals with bases $x_{1}+v x_{2}$, $x_{4}$, and $x_{2}, x_{3}-x_{4}$. So $K_{v, 0} \cong M^{8}$. This finishes the case where $s \neq 0$.

Now suppose that $s=0$. Then $D$ is equal to

$$
\left(\begin{array}{lll}
0 & t & 0 \\
u & 0 & 0 \\
0 & 0 & 0
\end{array}\right)
$$

If $u \neq 0$ then we can divide by it and obtain the derivation

$$
\left(\begin{array}{lll}
0 & a & 0 \\
1 & 0 & 0 \\
0 & 0 & 0
\end{array}\right)
$$

This leads to the Lie algebras

$$
M_{a}^{10}: \quad\left[x_{4}, x_{1}\right]=x_{2},\left[x_{4}, x_{2}\right]=a x_{1},\left[x_{3}, x_{1}\right]=x_{1},\left[x_{3}, x_{2}\right]=x_{2} .
$$

If the characteristic of $F$ is not 2 , then $M_{a}^{10} \cong M_{a-\frac{1}{4}}^{9}$. The isomorphism is given by $\phi\left(x_{1}\right)=2 y_{2}, \phi\left(x_{2}\right)=2 y_{1}-y_{2}, \phi\left(x_{3}\right)=y_{3}, \phi\left(x_{4}\right)=-\frac{1}{2} y_{3}+y_{4}$ (where the $x_{i}$ are the basis elements of $\left.M_{a}^{10}\right)$. Note that, if $a=0$, this gives an isomorphism with $N$. 
If the characteristic is 2 , then from a Gröbner basis computation it follows that $M_{a}^{10}$ is not isomorphic to $M_{b}^{9}$. So in this case we have a new series of Lie algebras. From a Gröbner basis computation we get that $M_{a}^{10} \cong M_{b}^{10}$ implies that $Y^{2}+X^{2} b+a=0$ is solvable in $F$, with $X \neq 0$. On the other hand, if $\beta$ and $\alpha \neq 0$ are such that $\beta^{2}+\alpha^{2} b+a=0$, then $\phi\left(x_{1}\right)=y_{1}, \phi\left(x_{2}\right)=\beta y_{1}+\alpha y_{2}, \phi\left(x_{3}\right)=y_{3}, \phi\left(x_{4}\right)=\beta y_{3}+\alpha y_{4}$ is an isomorphism. So $M_{a}^{10} \cong M_{b}^{10}$ if and only if $Y^{2}+X^{2} b+a=0$ has a solution in $F$, with $X \neq 0$. In particular, if the field is perfect (i.e., $F^{2}=F$ ) then $M_{a}^{10} \cong M_{0}^{10}$.

If $u=0$, and $t \neq 0$ then we divide by $t$. The corresponding Lie algebra has multiplication table

$$
\left[x_{4}, x_{2}\right]=x_{1},\left[x_{3}, x_{1}\right]=x_{1},\left[x_{3}, x_{2}\right]=x_{2} .
$$

If the characteristic is not 2 , then it is isomorphic to $N$, the isomorphism being $\phi\left(x_{1}\right)=$ $2 y_{1}-y_{2}, \phi\left(x_{2}\right)=y_{2}, \phi\left(x_{3}\right)=y_{3}, \phi\left(x_{4}\right)=-y_{3}+2 y_{4}$. If the characteristic is 2 , then this algebra is isomorphic to $M_{0}^{10}$, with in this case, $\phi\left(x_{1}\right)=y_{2}, \phi\left(x_{2}\right)=y_{1}, \phi\left(x_{3}\right)=y_{3}$, $\phi\left(x_{4}\right)=y_{4}$.

If $t=0$ as well, then the derivation is inner, and we obtain nothing new.

Now we consider the Lie algebra $K=L_{a}^{3}$. Its derivations consist of

$$
\left(\begin{array}{ccc}
u & a v & s \\
v & u+v & t \\
0 & 0 & 0
\end{array}\right)
$$

This means that, if $a \neq 0$, then modulo scalar factors there is only one outer derivation, namely

$$
D=\left(\begin{array}{lll}
1 & 0 & 0 \\
0 & 1 & 0 \\
0 & 0 & 0
\end{array}\right)
$$

leading to the Lie algebras

$$
\left[x_{4}, x_{1}\right]=x_{1},\left[x_{4}, x_{2}\right]=x_{2},\left[x_{3}, x_{1}\right]=x_{2},\left[x_{3}, x_{2}\right]=a x_{1}+x_{2} .
$$

However, by interchanging $x_{3}, x_{4}$ and $x_{1}, x_{2}$ we get the Lie algebra $K_{a}$ considered before (leading to the algebras $N, M^{8}, M_{a}^{9}$ ).

If $a=0$, then apart from the derivation above, we get two more:

$$
D_{1}=\left(\begin{array}{ccc}
1 & 0 & v \\
0 & 1 & 0 \\
0 & 0 & 0
\end{array}\right), \text { and } D_{2}=\left(\begin{array}{ccc}
0 & 0 & 1 \\
0 & 0 & 0 \\
0 & 0 & 0
\end{array}\right)
$$

Let $\sigma$ be the automorphism with matrix

$$
\left(\begin{array}{lll}
1 & 0 & v \\
0 & 1 & 0 \\
0 & 0 & 1
\end{array}\right)
$$


Then $\sigma D_{1} \sigma^{-1}$ is equal to $D$, so we get nothing new from $D_{1}$. However, we can not get rid of $D_{2}$ in this way. It leads to the Lie algebra

$$
\left[x_{4}, x_{3}\right]=x_{1},\left[x_{3}, x_{1}\right]=x_{2},\left[x_{3}, x_{2}\right]=x_{2} .
$$

But this is isomorphic to $M_{0,0}^{6}$, by $\phi\left(x_{1}\right)=y_{2}, \phi\left(x_{2}\right)=y_{3}, \phi\left(x_{3}\right)=y_{4}, \phi\left(x_{4}\right)=-y_{1}$.

Now we deal with the Lie algebra $K=L_{a}^{4}$. If $a \neq 0$ and the characteristic of $F$ is not 2 , then the derivations of $L_{a}^{4}$ are given by

$$
\left(\begin{array}{ccc}
u & a v & s \\
v & u & t \\
0 & 0 & 0
\end{array}\right) .
$$

Here modulo inner derivations, and scalar factors, there remains only one derivation

$$
\left(\begin{array}{lll}
1 & 0 & 0 \\
0 & 1 & 0 \\
0 & 0 & 0
\end{array}\right)
$$

leading to the Lie algebras

$$
\left[x_{4}, x_{1}\right]=x_{1},\left[x_{4}, x_{2}\right]=x_{2},\left[x_{3}, x_{1}\right]=x_{2},\left[x_{3}, x_{2}\right]=a x_{1} .
$$

This Lie algebra is isomorphic to $M_{a-\frac{1}{4}}^{9}$. The isomorphism is given by $\phi\left(x_{1}\right)=2 y_{2}, \phi\left(x_{2}\right)=$ $2 y_{1}-y_{2}, \phi\left(x_{3}\right)=-\frac{1}{2} y_{3}+y_{4}, \phi\left(x_{4}\right)=y_{3}$.

If $a \neq 0$ and the characteristic of $F$ is 2 , then the derivations are given by

$$
\left(\begin{array}{ccc}
u & a v & s \\
v & u+w & t \\
0 & 0 & w
\end{array}\right) .
$$

So modulo inner derivations we get

$$
\left(\begin{array}{ccc}
u & 0 & 0 \\
0 & u+w & 0 \\
0 & 0 & w
\end{array}\right) .
$$

If $w=0$ then this leads to the algebra that we have seen in the characteristic not 2 case. In this case it is isomorphic to $M_{a}^{10}$, by $\phi\left(x_{1}\right)=y_{1}, \phi\left(x_{2}\right)=y_{2}, \phi\left(x_{3}\right)=y_{4}, \phi\left(x_{4}\right)=y_{3}$.

If $u$ and $w$ are both nonzero, then after dividing we get the derivations

$$
\left(\begin{array}{ccc}
1 & 0 & 0 \\
0 & b & 0 \\
0 & 0 & 1+b
\end{array}\right) .
$$

Here we assume that $b \neq 1$ as we have already listed the corresponding algebra (it is isomorphic to $\left.M_{a}^{10}\right)$. The Lie algebras we now get are:

$$
M_{a, b}^{11}: \quad\left[x_{4}, x_{1}\right]=x_{1},\left[x_{4}, x_{2}\right]=b x_{2},\left[x_{4}, x_{3}\right]=(1+b) x_{3},\left[x_{3}, x_{1}\right]=x_{2},\left[x_{3}, x_{2}\right]=a x_{1} .
$$


(Here $a \neq 0, b \neq 1$.) Let $c \neq 0$ and $d \neq 1$. Set $\delta=(b+1) /(d+1)$. We claim that $M_{a, b}^{11} \cong M_{c, d}^{11}$ if and only if $\left(\delta^{2}+(b+1) \delta+b\right) / c$ and $a / c$ are squares in $F$. The only if part follows from inspection of the Gröbner basis. The if part from explicit construction of an isomorphism. Let $\gamma, \epsilon \in F$ be such that

$$
\gamma^{2}=\frac{1}{c}\left(\delta^{2}+(b+1) \delta+b\right), \text { and } \epsilon^{2}=\frac{a}{c} .
$$

If $\delta=1$ then $b=d$ and isomorphism follows already from the dimension 3 isomorphism. So we suppose that $\delta \neq 1$, and we set $\beta=\delta+1, \alpha=c \gamma$. Then $\phi: K_{a, b} \rightarrow K_{c, d}$ given by $\phi\left(x_{1}\right)=\alpha y_{1}+\beta y_{2}, \phi\left(x_{2}\right)=c \epsilon \beta y_{1}+\alpha \epsilon y_{2}, \phi\left(x_{3}\right)=\epsilon y_{3}, \phi\left(x_{4}\right)=\gamma y_{3}+\delta y_{4}$, is an isomorphism. In particular, if $F$ is perfect, then $M_{a, b}^{11} \cong M_{1,0}^{11}$.

If $w \neq 0$, but $u=0$, then we get the algebra

$$
\left[x_{4}, x_{2}\right]=x_{2},\left[x_{4}, x_{3}\right]=x_{3},\left[x_{3}, x_{1}\right]=x_{2},\left[x_{3}, x_{2}\right]=a x_{1} .
$$

If $a \neq 0$ and $a \neq 1$ then this is isomorphic to $M_{a, a}^{11}$, by $\phi\left(x_{1}\right)=y_{1}+a^{-1} y_{2}, \phi\left(x_{2}\right)=y_{1}+y_{2}$, $\phi\left(x_{3}\right)=y_{3}, \phi\left(x_{4}\right)=\frac{1}{a+1}\left(y_{3}+y_{4}\right)$. If $a=1$, then it is isomorphic to $M_{1,0}^{11}$, by $\phi\left(x_{1}\right)=y_{2}$, $\phi\left(x_{2}\right)=y_{1}, \phi\left(x_{3}\right)=y_{3}, \phi\left(x_{4}\right)=y_{4}$. If $a=0$, then it is isomorphic to $M_{0}^{10}$, by $\phi\left(x_{1}\right)=y_{4}$, $\phi\left(x_{2}\right)=y_{2}, \phi\left(x_{3}\right)=y_{1}, \phi\left(x_{4}\right)=y_{3}$.

Now suppose that $a=0$. Then the derivations (modulo inner derivations) are

$$
D=\left(\begin{array}{ccc}
u_{1} & 0 & v_{1} \\
0 & u_{1}+v_{3} & 0 \\
u_{3} & 0 & v_{3}
\end{array}\right)
$$

A general automorphism of $L_{0}^{4}$ is given by

$$
\phi=\left(\begin{array}{ccc}
\alpha_{1} & 0 & \gamma_{1} \\
\alpha_{2} & \alpha_{1} \gamma_{3}-\alpha_{3} \gamma_{1} & \gamma_{2} \\
\alpha_{3} & 0 & \gamma_{3}
\end{array}\right),
$$

where $\alpha_{1} \gamma_{3}-\alpha_{3} \gamma_{1} \neq 0$. Now the entry at position $(3,3)$ of $\left(\alpha_{1} \gamma_{3}-\alpha_{3} \gamma_{1}\right) \phi D \phi^{-1}$ is $-u_{1} \alpha_{3} \gamma_{1}-$ $u_{3} \gamma_{1} \gamma_{3}+v_{1} \alpha_{1} \alpha_{3}+v_{3} \alpha_{1} \gamma_{3}$. It is straightforward to see that, except in the case where $u_{1}=v_{3}=s \neq 0$ and $u_{3}=v_{1}=0$, we can choose the $\alpha_{i}, \gamma_{i}$ such that this becomes zero. In the former case we divide by $s$ and get the Lie algebra

$$
M^{12}: \quad\left[x_{4}, x_{1}\right]=x_{1},\left[x_{4}, x_{2}\right]=2 x_{2},\left[x_{4}, x_{3}\right]=x_{3},\left[x_{3}, x_{1}\right]=x_{2} .
$$

Otherwise $D$ is conjugate to

$$
D^{\prime}=\left(\begin{array}{ccc}
u_{1} & 0 & v_{1} \\
0 & u_{1} & 0 \\
u_{3} & 0 & 0
\end{array}\right)
$$

Now let $\phi$ be the automorphism given by the matrix

$$
\left(\begin{array}{ccc}
\alpha & 0 & 0 \\
0 & \alpha \beta & 0 \\
0 & 0 & \beta
\end{array}\right)
$$


where both $\alpha, \beta$ are nonzero. Then

$$
\phi D^{\prime} \phi^{-1}=\left(\begin{array}{ccc}
u_{1} & 0 & \frac{\alpha}{\beta} v_{1} \\
0 & u_{1} & 0 \\
\frac{\beta}{\alpha} u_{3} & 0 & 0
\end{array}\right) .
$$

First suppose that $u_{1} \neq 0$. Then we divide by it. If $v_{1}$ is also nonzero, then we choose $\alpha=u_{1}$ and $\beta=v_{1}$. This leads to the Lie algebras

$$
M_{a}^{13}: \quad\left[x_{4}, x_{1}\right]=x_{1}+a x_{3},\left[x_{4}, x_{2}\right]=x_{2},\left[x_{4}, x_{3}\right]=x_{1},\left[x_{3}, x_{1}\right]=x_{2} .
$$

From the Gröbner basis it follows that two of those algebras, with parameters $a$ and $b$, are isomorphic if and only if $a=b$.

Now suppose that $v_{1}=0$. If $u_{3} \neq 0$, then set $\alpha=u_{3}, \beta=u_{1}$. We get the Lie algebra

$$
\left[x_{4}, x_{1}\right]=x_{1}+x_{3},\left[x_{4}, x_{2}\right]=x_{2},\left[x_{3}, x_{1}\right]=x_{2} .
$$

If we set $\tilde{x}_{1}=x_{1}+x_{3}, \tilde{x}_{2}=-x_{2}, \tilde{x}_{3}=x_{1}, \tilde{x}_{4}=x_{4}$, then we see that with respect to this new basis the Lie algebra has the same multiplication table as $M_{0}^{13}$.

Suppose that $u_{3}$ is zero as well. Then we get the Lie algebra

$$
\left[x_{4}, x_{1}\right]=x_{1},\left[x_{4}, x_{2}\right]=x_{2},\left[x_{3}, x_{1}\right]=x_{2} .
$$

In this case we set $\tilde{x}_{1}=x_{1}, \tilde{x}_{2}=x_{2}, \tilde{x}_{3}=x_{1}+x_{3}, \tilde{x}_{4}=x_{4}$. Again we get the multiplication table of $M_{0}^{13}$.

Secondly, suppose that $u_{1}=0$. If $v_{1} \neq 0$, then we divide by it, and get the derivations

$$
\left(\begin{array}{lll}
0 & 0 & 1 \\
0 & 0 & 0 \\
a & 0 & 0
\end{array}\right)
$$

leading to the Lie algebras

$$
M_{a}^{14}: \quad\left[x_{4}, x_{1}\right]=a x_{3},\left[x_{4}, x_{3}\right]=x_{1},\left[x_{3}, x_{1}\right]=x_{2} .
$$

By setting $y_{i}=\alpha x_{i}$ for $i=1,2,4, y_{3}=x_{3}$, we see that this Lie algebra is isomorphic to the same one with parameter $\alpha^{2} a$. On the other hand, from a Gröbner basis computation we get that $M_{a}^{14} \cong M_{b}^{14}$ implies $a=\alpha^{2} b$ for some $\alpha$.

If $v_{1}=0$, then we get two more algebras. The first is a direct sum isomorphic to $M^{5}$. The other is

$$
\left[x_{4}, x_{1}\right]=x_{3},\left[x_{3}, x_{1}\right]=x_{2} .
$$

Here we set $\tilde{x}_{1}=x_{3}, \tilde{x}_{2}=-x_{2}, \tilde{x}_{3}=x_{1}, \tilde{x}_{4}=x_{4}$. This gives us the multiplication table of $M_{0}^{14}$.

There are some additional isomorphisms. If the characteristic of $F$ is not 2 , then $M_{0}^{13} \cong N$, by $\phi\left(x_{1}\right)=y_{1}+y_{2}, \phi\left(x_{2}\right)=3 y_{1}-\frac{3}{2} y_{2}, \phi\left(x_{3}\right)=y_{1}+y_{2}-y_{3}+2 y_{4}, \phi\left(x_{4}\right)=y_{3}$. 
For this reason we don't list $N$ separately (if the characteristic is not 2, then it doesn't exist).

Second, $M_{0,0}^{7} \cong M_{0}^{14}$, by $\phi\left(x_{1}\right)=-y_{4}, \phi\left(x_{2}\right)=y_{1}, \phi\left(x_{3}\right)=y_{2}, \phi\left(x_{4}\right)=y_{3}$.

If the characteristic is 2 , and $a=\alpha^{2} \neq 0$, then $M_{a}^{10} \cong M_{0}^{13}$, by $\phi\left(x_{1}\right)=y_{1}, \phi\left(x_{2}\right)=$ $\alpha y_{1}+\alpha y_{2}, \phi\left(x_{3}\right)=y_{1}+y_{2}+y_{4}, \phi\left(x_{4}\right)=\alpha y_{3}+\alpha y_{4}$. Note that if $a=0$ we also have isomorphism: $M_{0}^{10} \cong M_{1}^{10} \cong M_{0}^{13}$.

I have established the non-isomorphism of the remaining Lie algebras $M^{i}$ by Gröbner basis computations.

Remark. The Lie algebras $M_{0, b}^{11}$ do exist, and one may wonder where they occur in the list. We have $M_{0,0}^{11} \cong M^{12}$, and $M_{0, b}^{11} \cong M_{(b+1) / b^{2}}^{13}$ if $b \neq 0, b \neq 1$.

We count the number of solvable Lie algebras of dimension 4 over the finite field $\mathbb{F}_{q}$, where $q=p^{m}$. For that we start with a well-known lemma (see [1], Theorems 6.69, 6.695).

Lemma 4 Let $u \in \mathbb{F}_{q}$, where $q=2^{m}$. Then the equation $X^{2}+X+u$ has a solution in $\mathbb{F}_{q}$ if and only if

$$
\operatorname{Tr}_{2}(u)=\sum_{i=0}^{m-1} u^{2^{i}}=0 .
$$

The classes $M^{1}, M^{2}, M_{a}^{3}, M^{4}, M^{5}, M_{a, b}^{6}$ contain 1, 1, q, 1, 1, $q^{2}$ algebras respectively. As noted before, the class $M_{a, b}^{7}$ splits in three subclasses: $M_{a, a}^{7}, M_{a, 0}^{7}(a \neq 0)$, and $M_{0, b}^{7}$ $(b \neq 0)$. The first of these contains $q$ elements. We have $M_{a, 0}^{7} \cong M_{a^{\prime}, 0}^{7}$ if and only if $a=\alpha^{3} a^{\prime}$ for some $\alpha \in \mathbb{F}_{q}$. First suppose that $q$ is odd. If $q \equiv 1 \bmod 6$, then $X^{3}=1$ has 3 solutions in $\mathbb{F}_{q}$. In that case $\mathbb{F}_{q}$ contains $(q-1) / 3$ cubes and hence we get 3 algebras. If $q \not \equiv 1 \bmod 6$ then $X^{3}=1$ has 1 solution in $\mathbb{F}_{q}$ and hence $\mathbb{F}_{q}^{3}=\mathbb{F}_{q}$ and we get only 1 algebra. Now suppose that $p=2$, then $\operatorname{Tr}_{2}(1)=m$. So by Lemma $4, X^{2}+X+1$ has solutions in $\mathbb{F}_{q}$ if and only if $m$ is even. This is the same as saying that $p^{m} \equiv 4 \bmod 6$. In the same way as above we conclude that in this case $M_{a, 0}^{7}$ has 3 algebras. In the other case, $q \equiv 2 \bmod 6$, we get 1 algebra. The class $M_{0, b}^{7}$ contains 1 algebra if $p=2$, and 2 algebras if $p>2$. Summarising

$$
\left|M_{a, b}^{7}\right|= \begin{cases}q+5 & q \equiv 1 \bmod 6 \\ q+2 & q \equiv 2 \bmod 6 \\ q+3 & q \equiv 3 \bmod 6 \\ q+4 & q \equiv 4 \bmod 6 \\ q+3 & q \equiv 5 \bmod 6\end{cases}
$$

From $M^{8}$ we get 1 algebra. Now we consider $M_{a}^{9}$. First suppose that $q$ is odd. We have to find the set of $a \in \mathbb{F}_{q}$ such that $T^{2}-T-a$ has no root in $\mathbb{F}_{q}$. Suppose that this equation has a root $\alpha$. Then the other root is $1-\alpha$ and $a=\alpha^{2}-\alpha$. Let $B$ be the set of all $\alpha^{2}-\alpha$ for $\alpha \in \mathbb{F}_{q}$. If the equation $X^{2}-X=c$ has one solution in $\mathbb{F}_{q}$, then it has two solutions, unless $c=-\frac{1}{4}$. This implies that $|B|=(q+1) / 2$. Let $A$ be the set of all $a \in \mathbb{F}_{q}$ such that $T^{2}-T-a$ has no root in $\mathbb{F}_{q}$. Then $|A|=(q-1) / 2$. Also, for $0 \neq \beta \in \mathbb{F}_{q}$ we define 
$h_{\beta}: \mathbb{F}_{q} \rightarrow \mathbb{F}_{q}$ by $h_{\beta}(x)=\beta^{2}\left(x+\frac{1}{4}\right)-\frac{1}{4}$. Then $h_{\beta}$ is a bijection. It stabilizes $B$ and hence $A$. Now $M_{a}^{9} \cong M_{b}^{9}$ precisely if $h_{\beta}(a)=b$ for some $0 \neq \beta \in \mathbb{F}_{q}$. There are exactly $(q-1) / 2$ different $h_{\beta}$ 's. So all $M_{a}^{9}$ for $a \in A$ are isomorphic. Hence we get 1 algebra. Secondly, suppose that $q$ is even. Choose $a, b$ such that $T^{2}+T+a$ and $T^{2}+T+b$ have no roots in $\mathbb{F}_{q}$. Then by Lemma 4. $\operatorname{Tr}_{2}(a)=\operatorname{Tr}_{2}(b)=1$. But then $\operatorname{Tr}_{2}(a+b)=0$ and $T^{2}+T+a+b$ has roots in $\mathbb{F}_{q}$. Hence $M_{a}^{9} \cong M_{b}^{9}$. So we get one algebra in this case as well.

The classes $M_{a}^{10}$ and $M_{a, b}^{11}$ are only defined for characteristic 2. For perfect fields they both have one algebra. However, the algebra in $M_{a}^{10}$ disappears due to the isomorphism with $M_{0}^{13}$.

The classes $M^{12}$ and $M_{a}^{13}$ have 1 and $q$ algebras respectively. For $M_{a}^{14}$ we exclude $a=0$, as that algebra is isomorphic to $M_{0,0}^{7}$. Therefore this class contains 1 algebra if $p=2$ and 2 algebras if $p>2$.

Now we add these numbers, and find that the total number of solvable Lie algebras over $\mathbb{F}_{q}$ is

$$
q^{2}+3 q+9+\left\{\begin{array}{cc}
5 & q \equiv 1 \bmod 6 \\
2 & q \equiv 2 \bmod 6 \\
3 & q \equiv 3 \bmod 6 \\
4 & q \equiv 4 \bmod 6 \\
3 & q \equiv 5 \bmod 6
\end{array}\right.
$$

which is slightly more than the number found in [10].

Remark. With $L_{4, i}$ as in [10] we have $L_{4,1} \cong M^{1}, L_{4,2} \cong M_{5}$ (for this one has to correct the table given in [10]; with the table as given in [10], we have $\left.L_{4,2} \cong L_{4,3}\right), L_{4,3} \cong M_{0,0}^{7}$, $L_{4,4} \cong M^{4}, L_{4,5} \cong M_{0}^{3}, L_{4,6} \cong M_{0, \alpha}^{7}, L_{4,7} \cong M_{0,-\alpha}^{6}, L_{4,8} \cong M^{8}, L_{4,9} \cong M_{0,-\frac{1}{4}}^{6}$ (if the characteristic is not 2 ), $L_{4,9} \cong M_{0,1}^{7}$ (if the characteristic is 2 ), $L_{4,10} \cong M^{2}, L_{4,11} \cong M_{\alpha}^{3}$, $L_{4,12} \cong M_{\alpha_{3},-\alpha_{2}}^{6}, L_{4,13} \cong M_{\alpha, \alpha}^{7}, L_{4,14} \cong M_{\alpha, 0}^{7}, L_{4,8}^{\prime} \cong M_{-\alpha}^{9}, L_{4,15} \cong M_{-2,3}^{7}$ (characteristic not 3) $L_{4,15} \cong M_{1}^{3}$ (characteristic 3$), L_{4,16} \cong M_{-2 \alpha^{3}+\alpha^{2}, 3 \alpha^{2}-2 \alpha}^{6}\left(\alpha \neq \frac{1}{3}\right), L_{4,16} \cong M_{1}^{3}\left(\alpha=\frac{1}{3}\right)$, $L_{4,17} \cong M_{\frac{1}{27},-\frac{1}{3}}^{6}$ (characteristic not 3 and $\left.\alpha \neq 0\right), L_{4,17} \cong M_{1,0}^{7}$ (characteristic 3 , and $\left.\alpha \neq 0\right)$, $L_{4,17} \cong M_{0,0}^{7}(\alpha=0), L_{4,18} \cong M^{12}, L_{4,19} \cong M_{\alpha}^{14}, L_{4,20} \cong M_{-\alpha}^{13}$.

In [10] the algebra $M_{a, b}^{11}$ is missing. This can be explained by the circumstance that the method used in [10] relies on the derived algebra being nilpotent. Now, if $a \neq 0$ and $b \neq 1$ then the derived algebra of $M_{a, b}^{11}$ is not nilpotent.

Remark. Of course the next step will be to describe the classification for dimension 5 . This will be the subject of a next paper. A problem that may occur is that the computation of the coordinates of the elements of a Gröbner basis (with respect to the input basis) makes the algorithm rather slow. So there may occur cases where it is impossible to compute a Gröbner basis (with coordinates).

Remark. As in the dimension 3 case, it is possible to formulate an algorithm that for a given solvable Lie algebra $K$ of dimension 4 finds the $M^{i}$ to which it is isomorphic. First we find a 3-dimensional ideal, and establish to which of the $L^{i}$ it is isomorphic. Then for 
each of the four possibilities we basically follow the classification procedure.

\section{References}

[1] E. R. Berlekamp. Algebraic coding theory. McGraw-Hill, New York, 1968.

[2] Computational Algebra Group, School of Mathematics and Statistics University of Sydney, Australia. The Magma System for Algebra, Number Theory and Geometry.

[3] D. Cox, J. Little, and D. O'Shea. Ideals, Varieties and Algorithms: An Introduction to Computational Algebraic Geometry and Commutative Algebra. Springer Verlag, New York, Heidelberg, Berlin, 1992.

[4] V. P. Gerdt and W. Lassner. Isomorphism verification for complex and real Lie algebras by Gröbner basis technique. In N. H. Ibragimov et al., editor, Modern Group Analysis: Advanced Analytical and Computational Methods in Mathematical Physics, pages 245-254, Dordrecht, 1993. Kluwer Academic Publishers.

[5] W. A. de Graaf. Lie Algebras: Theory and Algorithms, volume 56 of North-Holland Mathematical Library. Elsevier Science, 2000.

[6] B. Hartley and T. O. Hawkes. Rings, modules and linear algebra. A further course in algebra describing the structure of Abelian groups and canonical forms of matrices through the study of rings and modules. Chapman and Hall Ltd., London, 1970.

[7] G. M. Mubarakzjanov. Classification of real structures of Lie algebras of fifth order. Izv. Vysš. Učebn. Zaved. Matematika, 1963(3 (34)):99-106, 1963. (Russian).

[8] G. M. Mubarakzjanov. Classification of solvable Lie algebras of sixth order with a nonnilpotent basis element. Izv. Vysš. Učebn. Zaved. Matematika, 1963(4 (35)):104-116, 1963. (Russian).

[9] J. Patera, R. T. Sharp, P. Winternitz, and H. Zassenhaus. Invariants of real low dimensional Lie algebras. Journal of Mathematical Physics, 17:986-994, 1976.

[10] J. Patera and H. Zassenhaus. Solvable Lie algebras of dimension $\leq 4$ over perfect fields. Linear Algebra Appl., 142:1-17, 1990.

[11] D. Rand, P. Winternitz, and H. Zassenhaus. On the identification of a Lie algebra given by its structure constants. I. Direct decompositions, Levi decompositions and nilradicals. Linear Algebra Appl., 109:197-246, 1988. 